\title{
The British Neurosurgical Trainee Research Collaborative: Five years on
}

\author{
Aswin Chari ${ }^{1}$ - Aimun A. Jamjoom ${ }^{2}$ • Ellie Edlmann ${ }^{3}$ - Aminul I. Ahmed ${ }^{4}$. \\ Ian C. Coulter ${ }^{5}$. Ruichong $\mathrm{Ma}^{6} \cdot$ Paul May $^{7} \cdot$ Paul M. Brennan ${ }^{2}$. \\ Peter J. A. Hutchinson ${ }^{3,8}$ • Angelos G. Kolias ${ }^{3,8}$ • British Neurosurgical Trainee Research \\ Collaborative
}

Received: 28 September 2017 / Accepted: 2 October 2017 /Published online: 4 November 2017

(C) The Author(s) 2017. This article is an open access publication

\begin{abstract}
Since its inception in 2012, the British Neurosurgical Trainee Research Collaborative (BNTRC) has established itself as a robust example of a trainee-led research collaborative. This article summarises the work of the collaborative over its first 5 years of existence, outlining the structure, its research projects, impact and future directions.
\end{abstract}

Keywords Collaborative $\cdot$ Research $\cdot$ Clinical trials . Resident · Trainee

\section{Introduction}

Multi-centre research can limit bias and can increase the external validity of study results. The British Neurosurgical Trainee Research Collaborative (BNTRC) was founded in

Angelos G. Kolias

angeloskolias@gmail.com

1 Department of Neurosurgery, Royal London Hospital, London, UK

2 Division of Neurosurgery, Department of Clinical Neuroscience, Western General Hospital, Edinburgh, UK

3 Division of Neurosurgery, Department of Clinical Neurosciences, Addenbrooke's Hospital and University of Cambridge, Cambridge CB2 0QQ, UK

4 Department of Neurosurgery, Wessex Neurological Centre and University of Southampton, Southampton, UK

5 Department of Neurosurgery, Royal Victoria Infirmary, Newcastle, UK

6 Department of Neurosurgery, John Radcliffe Hospital, Oxford, UK

7 Department of Neurosurgery, The Walton Centre, Liverpool, UK

8 Surgery Theme, Cambridge Clinical Trials Unit, Cambridge Biomedical Campus, Cambridge, UK
2012, with the aim of encouraging high-quality, multi-centre research and fostering a culture of collaboration. This includes engaging different units, increasing the accessibility of research for neurosurgical trainees and consultants and, ultimately, improving care and outcomes for neurosurgical patients. It was established as an initiative of the British Neurosurgical Trainee Association (BNTA) with the strong support of the Society of British Neurological Surgeons (SBNS) [14].

The BNTRC was inspired by the successes of the West Midlands Research Collaborative (WMRC), a regional general surgical research collaborative established 4 years prior. The WMRC had demonstrated significant success in implementing a trainee-led collaborative, including funding and running a randomised clinical trial that recruited to its target ahead of schedule [23].

The inaugural meeting was held at the Royal College of Surgeons of England (RCS) on 19 October 2012, with trainees from 13 of the 18 training programmes in the UK and Ireland in attendance and representatives from the BNTA and SBNS. Several projects were proposed by the attending trainees and a decision was made to pursue three projects (Table 1). Specific focus was placed on the national audit of chronic subdural haematoma (CSDH) as it was a topic relevant to trainees, and the prospective observational study model would serve as a proof of principle in terms of establishing the BNTRC network. Following the meeting, the SBNS agreed to support the BNTRC with a $£ 10,000$ starter grant to help with expenses [15].

\section{Structure and research model}

The formal structure of the BNTRC was established at an open meeting in January 2015, where a constitution was 
Table 1 Studies selected for implementation at the inaugural BNTRC meeting in October 2012

Studies proposed at the inaugural BNTRC meeting

A national audit of chronic subdural haematoma (CSDH)

A national audit of cranioplasty

A randomised trial of craniotomy vs craniectomy for acute subdural haematoma (RESCUE-ASDH)

established [4]. Membership was opened to all junior doctors working in UK neurosurgery and the committee structure, involving the study leads and representatives from the BNTA, was upheld (Fig. 1).

The research model is based upon established trainee research collaborative models with each study having trainee leads (one to two members), a steering committee (five to six members) and local collaborators at each unit $[1,2]$. The steering committee, which comprises of trainees and consultants, has responsibility for study design, execution, data integrity, analysis and write-up, with local collaborators being responsible for accurate data collection within each unit (Fig. 1). Although a general model, this is adapted for each study and is agreed in advance, in line with the constitution.

Protocols for each study are written by the study leads with input from the steering committee and are published (either online on our website or in a peer-reviewed journal) prior to study commencement. Each local lead is responsible for gaining relevant regulatory approvals (local audit, clinical governance or ethical approvals) and ensuring robust data collection. Once the data collection period is complete, the study leads and steering committee are responsible for data cleansing and analysis.

A key aspect of each study is the data management strategy. This includes finding robust platforms for data collection. The BNTRC has explored a number of strategies, from the simple method of combining anonymised data on Microsoft Excel to robust online platforms for data entry such as the Outcomes Registry Intervention and Operation Network (http:/www. orioncloud.org) and Castor Electronic Data Capture (http:// www.castoredc.com). Each have their advantages and disadvantages, and the best platform for each project must be individually chosen.

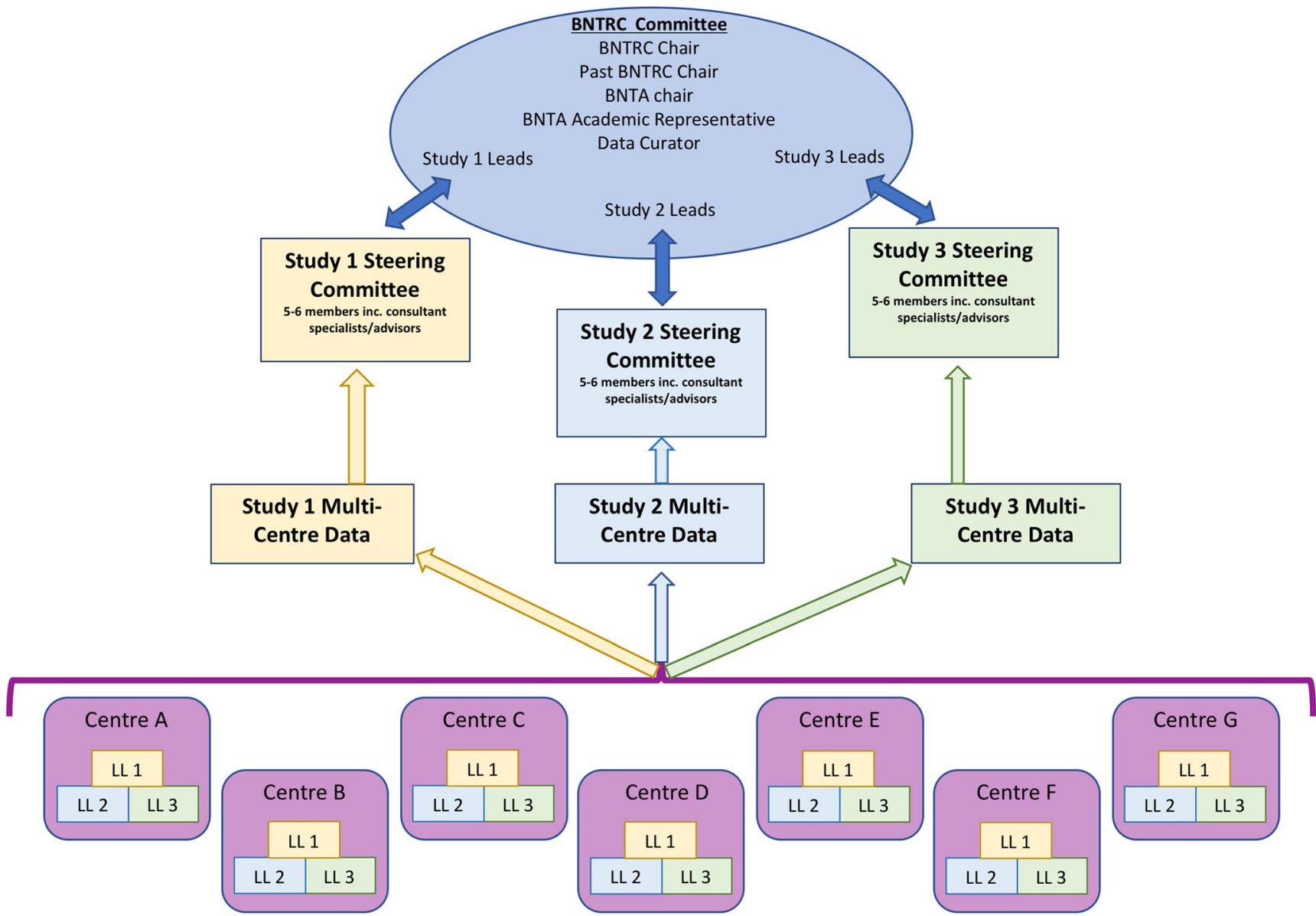

Fig. 1 Collaborative research model used by the BNTRC. The BNTRC committee is composed of the individual study leads and representatives from the BNTA. Each study has a study steering committee and local leads $(L L)$ at each neurosurgical centre 
The prospectively agreed writing group, consisting of the study leads, steering committee and selected centre leads, are then responsible for drafting the manuscript, which is then sent to all collaborators prior to submission. Authorship criteria are broadly defined in the BNTRC constitution and each study steering committee also prospectively agrees the specifics of its own policy. The BNTRC is always the final author with all contributors acknowledged as PubMed-citable collaborators in published manuscripts. Following publication of the initial study manuscript, it was also agreed that the data would be made available to BNTRC members for further analyses, in a structured way [4].

\section{Research projects}

Since 2012, the BNTRC has been involved with ten projects (four complete, six ongoing), with several new projects in the pipeline (Table 2). Project proposals are submitted through open calls to the entire UK neurosurgical trainee population (via established BNTA mailing lists). The proposals then undergo review by both the BNTRC committee and representatives of the SBNS academic committee, being assessed for the importance of the scientific question and the relative benefit of running a multi-centre study via the BNTRC. Whilst we initially started focusing on one project at a time, the collaborative has progressed to running multiple simultaneous projects.

The two randomised controlled trials, the Dex-CSDH and Rescue-ASDH trials (Table 2), have attracted funding from the UK National Institute of Health Research (NIHR) and are supported by the Cambridge Clinical Trials Unit. Although run by consultant-level investigators, the BNTRC and its members have had a crucial role in protocol development, funding applications and continue to play vital roles in patient recruitment and trial delivery. Collectively, these studies have randomised more than 600 patients in the last 3 years, illustrating the feasibility of trainee collaborative-led trial delivery.

\section{Communications, website and social media}

Communication and raising awareness about the BNTRC and its projects is a central strategy of the collaborative. To achieve this, the BNTRC has developed a website, produces a regular newsletter and is active on social media outlets such as Twitter.

In a bid to be as inclusive as possible, the BNTRC sends all communications via the established BNTA mailing list, which is open to all non-consultant doctors working in neurosurgery in the UK. Additional communications (such as newsletters) are distributed more widely, including to the SBNS and European Association of Neurosurgical Societies (EANS).
A BNTRC website was also developed to act as a resource for trainees and interested parties to learn more about the collaborative. It contains a wealth of information on the structure of the group, its constitution and the range of BNTRC projects. It is an important route for communication from interested doctors and medical students. Regular communications are also sent out via the BNTRC Twitter account (@BNTRC), which acts as an important social media resource for the collaborative. The group has an active account with over 650 followers and 9600 impressions over a 3-month period (FebApr 2017). It is a useful means to communicate news and progress reports about BNTRC projects.

\section{Impact: benefits for all stakeholders}

The most important stakeholders in collaborative research are patients, who stand to benefit through the comprehensive, high-quality investigation of clinically relevant questions. Over the last 5 years, the projects completed by the BNTRC have demonstrated the ability to benefit patients, in terms of identifying practices associated with improved outcomes. For example, the National CSDH audit identified that practices associated with unfavourable outcomes included lack of post-operative drain, use of post-operative bed rest and single burr-hole drainage [3].

Neurosurgical trainees also stand to benefit from involvement with the BNTRC. Trainees get the opportunity to be involved in the development and execution of robustly designed research protocols, skills that are vital in inculcating a generation of academically equipped neurosurgical consultants of the future. We also hope to contribute positively to the high-calibre research output of the UK neurosurgical community, which has produced many recent landmark clinical trials, including the STICH trials, RESCUEicp and STASH [7, 11, 18, 19].

From a national perspective, the BNTRC is viewed as one of the flagship specialty surgery research collaboratives, both in terms of the extent of its research activities and output so far $[10,20]$. Importantly, the network has engaged trainees from all UK neurosurgical units (Table 2). It has also played a key role in the National Research Collaborative (NRC), the umbrella body for UK surgical research collaboratives. It co-hosted the 2016 NRC Meeting, at the Royal College of Surgeons of England (London) in November 2016, along with the Reconstructive Surgery Trials Network (RSTN), the plastic surgery collaborative. It has also played a key role in shaping NRC policy, in terms of recognising and rewarding collaborative research and bolstering the infrastructure of trainee-led research collaboratives [16].

As the first of its kind in the global neurosurgical community, the BNTRC has played a pivotal role in setting an 


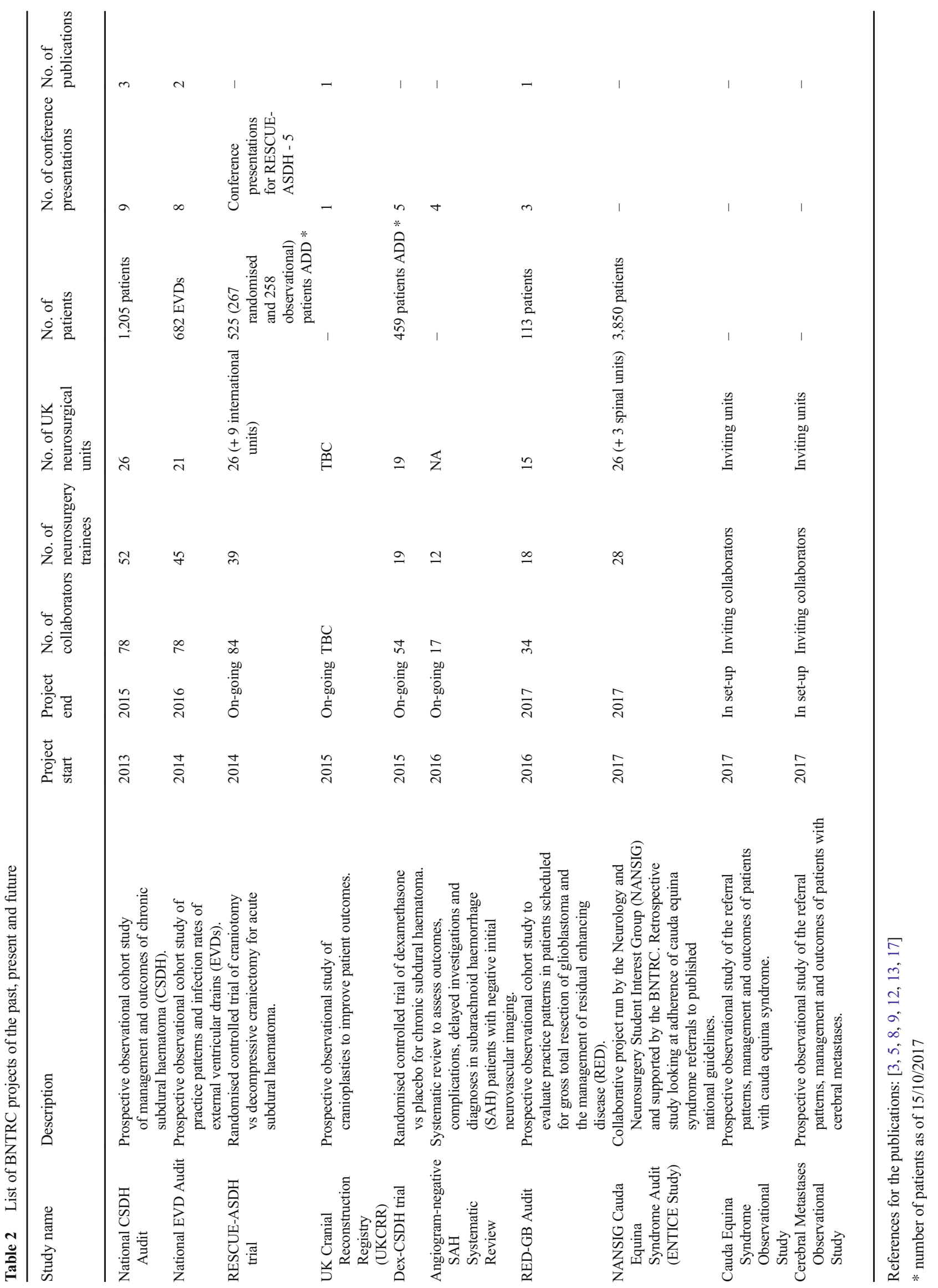


example to trainee neurosurgeons around the world. The collaborative has served as an inspiration and has provided support to a number of other collaboratives, including the Canadian Neurosurgical Research Collaborative (CNRC) [24], the Dutch Neurosurgical Trainee Research Network (DNTRN) and a new Scandinavian collaborative that is at its inception.

\section{Challenges}

Over the last 5 years, the collaborative has faced a number of challenges. We have addressed some of these in this section, which, along with a previously published 'how to' guide [6], should act as a useful resource to trainees looking to establish their own research collaboratives.

At inception, the biggest challenges include selling the model to trainees, consultants and supporting professional bodies. The BNTRC has been fortunate in having the unwavering support of the SBNS and the SBNS academic committee. Examples of successful trainee research collaboratives, such as the WMRC, were instrumental in convincing the trainee community of the potential of the collaborative research model.

The choice and success of the first project is also crucial to convincing all the stakeholders of the potential of the collaborative. It is prudent to pick a simple, noncontroversial research question, with an observational study design that will serve as a conduit for establishing the multi-centre network. The study should have a relatively short timescale, with clearly established author and collaborator policies, rewarding all collaborators for their involvement.

Each project also requires dedicated and motivated project leads and steering committee members, who are willing to drive the project forwards. Key challenges for the committee include developing a watertight protocol, obtaining ethical approvals and assembling the team of collaborators at each centre. In the UK, approvals are facilitated by having separate approval processes for 'audit' projects, which are less stringent than the ethical approval process for research projects. Data collection platforms also require careful thought, taking into account ease of use, financial considerations and security of the platform. As outlined above, the BNTRC has used a number of different platforms and we feel this flexibility has educated the group about the pros and cons of each, allowing us to tailor the choice of platform based on the particular needs of future projects.

Although the BNTRC established their constitution 3 years into its existence, we would advocate new groups establish a constitution at inception, outlining transparent policies for authorship, leadership and choice of new projects.

\section{Future directions}

In addition to completing and continuing to expand the current portfolio of projects, there are several challenges that lie ahead. First and foremost is sustaining enthusiasm and interest in collaborative research, and this can only be achieved by ensuring that the collaborative projects are relevant to our specialty. Enhancing our relationship with the SBNS and each of the subspecialty groups (e.g. trauma, vascular, CSF, oncology, spinal, etc.) will facilitate this. Second, maintaining the highest standards when conducting audit and research will be critical. Following relevant guidance, such as the IDEAL framework, will be helpful in that respect. The IDEAL framework describes the research stages from first-in-human to evaluation in randomised trials (www.ideal-collaboration. net) [22]. Third, the collaborative projects rely on the voluntary contributions (time and effort) of trainees; this means that their contribution needs to be fairly recognised and rewarded. Moreover, as senior trainees qualify, a role for them should be found within the network, as their experience will be valuable. Fourth, trials and studies enrolling patients in the non-emergency setting are clearly important in neurosurgery and we believe that the BNTRC could be successful in that respect too. However, this assumption will need to be tested in the future as studies in the non-emergency setting have their own set of challenges (e.g. strong surgeon and patient preferences). Other specialty trainees, including neurologists, radiologists and anaesthetists should be engaged in cross-specialty projects that will be crucial to improving patient care too. Finally, trainee collaboration at a global level is an exciting opportunity for enhancing the relevance of research findings for even more patients. The BNTRC remains committed to assisting trainees interested in setting up collaboratives in their countries with practical advice and support. The trainee collaborative model, at a global level, can contribute to the infrastructure networks required to increase access to neurosurgical services in low- and middle-income countries [21].

\section{Conclusions}

In the 5 years since its inception in 2012, the BNTRC has come a long way. There are significant challenges that lie ahead in sustaining and expanding upon this early success, with much of it depending on the sustained enthusiasm of future generations of UK neurosurgical trainees.

Acknowledgements The BNTRC would like to thank the Society of British Neurological Surgeons (SBNS) and the SBNS Academic Committee for their unwavering support and the initial seed funding. We would also like to thank the Surgical Trials Initiative of the Royal College of Surgeons of England. Peter Hutchinson is supported by a Research Professorship from the National Institute for Health Research 
(NIHR), the NIHR Cambridge Biomedical Research Centre, a European Union Seventh Framework Program grant (CENTER-TBI; grant no. 602150), and the Royal College of Surgeons of England. Angelos Kolias is supported by a Clinical Lectureship, School of Clinical Medicine, University of Cambridge and is the neurosurgical specialty lead for the IDEAL Collaboration (www.ideal-collaboration.net).

Open Access This article is distributed under the terms of the Creative Commons Attribution 4.0 International License (http:// creativecommons.org/licenses/by/4.0/), which permits unrestricted use, distribution, and reproduction in any medium, provided you give appropriate credit to the original author(s) and the source, provide a link to the Creative Commons license, and indicate if changes were made.

\section{References}

1. Bhangu A, Fitzgerald JE, Kolias AG (2014) Trainee-led research collaboratives: a novel model for delivering multi-centre studies. ANZ J Surg 84:902-903

2. Bhangu A, Kolias AG, Pinkney T, Hall NJ, Fitzgerald JE (2013) Surgical research collaboratives in the UK. Lancet 382:1091-1092

3. Brennan PM, Kolias AG, Joannides AJ, Shapey J, Marcus HJ, Gregson BA, Grover PJ, Hutchinson PJ, Coulter IC, British Neurosurgical Trainee Research Collaborative (2017) The management and outcome for patients with chronic subdural hematoma: a prospective, multicenter, observational cohort study in the United Kingdom. J Neurosurg 127:732-739

4. British Neurosurgical Trainee Research Collaborative (2015) BNTRC Constitution. https://docs.wixstatic.com/ugd/ff5354 85ddd6533bf64f8bad64cbeec1f68816.pdf. 2017

5. Coulter IC, Kolias AG, Marcus HJ, Ahmed AI, Alli S, Al-Mahfoudh R, Borg A, Cowie CJ, Hill CS, Joannides AJ, Jones TL, Kailaya-Vasan A, Livermore JL, Narayanamurthy H, Ngoga D, Shapey J, Tarnaris A, Gregson BA, Gray WP, Nelson RJ, Hutchinson PJ, Brennan PM, UK Neurosurgical Research Network, British Neurosurgical Trainee Research Collaborative (2014) Proposal for a prospective multi-centre audit of chronic subdural haematoma management in the United Kingdom and Ireland. Br J Neurosurg 28:199-203

6. Dowswell G, BartlettDC, FutabaK, WhiskerL, Pinkney TD(2014)How to set up and manage a trainee-led research collaborative. BMC MedEdu 14:94

7. Hutchinson PJ, Kolias AG, Timofeev IS, Corteen EA, Czosnyka M, Timothy J, Anderson I, Bulters DO, Belli A, Eynon CA, Wadley J, Mendelow AD, Mitchell PM, Wilson MH, Critchley G, Sahuquillo J, Unterberg A, Servadei F, Teasdale GM, Pickard JD, Menon DK, Murray GD, Kirkpatrick PJ, RESCUEicp Trial Collaborators (2016) Trial of Decompressive Craniectomy for traumatic intracranial hypertension. N Engl J Med 375:1119-1130

8. Jamjoom AA, Joannides A, Poon M, Chari A, Zaben M, Abdulla MAH, Roach J, Glancz LJ, Sloth A, Duddy J, Brennan PM, Bayston R, Bulters DO, Mallucci CL, Jenkinson MD, Gray WP, Kandasamy J, Hutchinson PJ, Kolias AG, Ahmed AI, British Neurosurgical Trainee Research Collaborative (2017) A prospective, multi-centre study of external ventricular drainage-related infections in the United Kingdom and Ireland. J Neurol Neurosurg Psychiatry.

9. Jamjoom AA, Kolias AG, Zaben M, Chari A, Kitchen J, Joannides A, Brennan PM, Kandasamy J, Gatscher S, Gray WP, Jenkinson MD, Bulters DO, Mallucci CL, Schuster H, Hutchinson PJ, Ahmed AI, UK Neurosurgical Research Network, British Neurosurgical Trainee Research Collaborative (2014) External ventricular drainage: is it time to look at national practice? Br J Neurosurg 4:1-2

10. Jamjoom AA, Phan PN, Hutchinson PJ, Kolias AG (2016) Surgical trainee research collaboratives in the UK: an observational study of research activity and publication productivity. BMJ Open 6: e010374

11. Kirkpatrick PJ, Turner CL, Smith C, Hutchinson PJ, Murray GD (2014) Simvastatin in aneurysmal subarachnoid haemorrhage (STASH): a multicentre randomised phase 3 trial. Lancet Neurol 13:666-675

12. Kolias AG, Bulters DO, Cowie CJ, Wilson MH, Afshari FT, Helmy A, Broughton E, Joannides AJ, Zebian B, Harrisson SE, Hill CS, Ahmed AI, BaroneDG, ThakurB,McMahonCJ,AdlamDM,BentleyRP, ToliasCM, Mitchell PM, Whitfield PC, Critchley GR, Belli A, Brennan PM, Hutchinson PJ(2014) Proposal for establishment of the UK cranial reconstruction registry (UKCRR). Br J Neurosurg 28:310-314

13. Kolias AG, CoulterIC,MarcusHJ, GrayWP,BrennanPM,HutchinsonPJ (2014) Chronic subdural haematoma: how can we improve patient care and outcomes? Br J Neurosurg 28:136-137

14. Kolias AG, Cowie CJ, Tarnaris A, Hutchinson PJ, Brennan PM (2012) Proposal for a British neurosurgical trainee research collaborative. Br J Neurosurg 26:434-435

15. KoliasAG,JonesTL,CowieCJ,CoulterIC,AfshariFT, TarnarisA,Nelson RJ, Gray WP, Hutchinson PJ, Brennan PM(2013) A report from the inaugural meeting of the British neurosurgical trainee research collaborative held in the Royal College of Surgeons of England, 19 October 2012. Br J Neurosurg 27:307-310

16. LeeMJ,BhanguA,BlencoweNS,NepogodievD, GokaniVJ,HarriesRL, Akinfala M, Ali O, Allum W, Bosanquet DC, Boyce K, Bradburn M, Chapman SJ, Christopher E, Coulter I, Dean BJ, Dickfos M, El Boghdady M, Elmasry M, Fleming S, Glasbey J, Healy C, Kasivisvanathan V, Khan KS, Kolias AG, Lee SM, Morton D, O'Beirne J, Sinclair P, Sutton PA (2016) Academic requirements for certificate of completion of training in surgical training: consensus recommendations from the Association of Surgeons in training/National Research Collaborative Consensus Group. IntJ Surg 36(Suppl 1):S24-S30

17. Ma R, Chari A, Brennan PM, Alalade A, Anderson I, Sloth A, Marcus HJ, Watts C, British Neurosurgical Trainee Research Collaborative (2017) Residual enhancing disease after surgery for glioblastoma: evaluation of practice in the United Kingdom. NeuroOncol Pract. https://doi.org/10.1093/nop/npx023

18. Mendelow AD, Gregson BA, Fernandes HM, Murray GD, Teasdale GM, Hope DT, Karimi A, Shaw MD, Barer DH (2005) Early surgery versus initial conservative treatment in patients with spontaneous supratentorial intracerebral haematomas in the International Surgical Trial in Intracerebral Haemorrhage (STICH): a randomised trial. Lancet 365:387-397

19. Mendelow AD, Gregson BA, Rowan EN, Murray GD, Gholkar A, Mitchell PM (2013) Early surgery versus initial conservative treatment in patients with spontaneous supratentorial lobar intracerebral haematomas (STICH II): a randomised trial. Lancet 382:397-408

20. Nepogodiev D, Chapman SJ, Kolias AG, Fitzgerald JE, Lee M, Blencowe NS (2017) The effect of trainee research collaboratives in the UK. Lancet Gastroenterol Hepatol 2:247-248

21. Park KB, Johnson WD, Dempsey RJ (2016) Global neurosurgery: the unmet need. World Neurosurg 88:32-35

22. Pennell CP, Hirst AD, Campbell WB, Sood A, Agha RA, Barkun JS, McCulloch P (2016) Practical guide to the idea, development and exploration stages of the IDEAL framework and recommendations. Br J Surg 103:607-615

23. Pinkney TD, Calvert M, Bartlett DC, Gheorghe A, Redman V, Dowswell G, Hawkins W, Mak T, Youssef H, Richardson C, Hornby S, Magill L, Haslop R, Wilson S, Morton D (2013) Impact of wound edge protection devices on surgical site infection after laparotomy: multicentre randomised controlled trial (ROSSINI Trial). BMJ 347: $\mathrm{f} 4305$

24. Tso MK, Dakson A, Ahmed SU, Bigder M, Elliott C, Guha D, IorioMorin C, Kameda-Smith M, Lavergne P, Makarenko S, Taccone MS, Wang B, Winkler-Schwartz A, Sankar T, Christie SD (2017) Operative landscape at Canadian neurosurgery residency programs. Can J Neurol Sci 44:415-419 